Pacific Journal of Mathematics

INTEGRAL INVARIANTS FOR VECTORS OVER LOCAL 


\title{
INTEGRAL INVARIANTS FOR VECTORS OVER LOCAL FIELDS
}

\author{
D. G. JAMES
}

This paper considers isometric invariants of vectors in lattices (quadratic forms) over the ring of integers in a local field for the prime 2. By extending the notion of order to vectors in the lattice we obtain a set of invariants which enable the general vector to be decomposed into a sum of simple vectors. The lengths of these simple vectors are invariant modulo certain powers of 2 and these lengths together with the original invariants form a complete set for the 2-adic integers. In the special case where there are no one dimensional, orthogonal sublattices (improper quadratic forms) the invariants form a complete set for all local fields.

Let $F$ be a local field, that is a field complete with respect to a discrete, non-archimedean valuation with a finite residue class field, and $R$ the ring of integers in $F$. Denote by $\nu(x)$ the order of the element $x$ in $F$ and by $\pi$ a fixed prime in $R$; we may assume that $\nu(\pi)=1$. Let $V$ be a nonsingular lattice over $R$, i.e. a finite dimensional, torsion-free $R$-module with scalar product $\alpha \cdot \beta \in R$. An isometry $\varphi$ of $V$ is a one-to-one, linear transformation of $V$ satisfying $\varphi(\alpha) \cdot \varphi(\beta)=\alpha \cdot \beta$. We investigate the isometric invariants of vectors $\alpha \in V$ in the unramified dyadic case, $\nu(2)=1$. The $p$-adic case ( $p$ odd) has been considered by Rosenzweig [7] and an alternative approach by Ankeny (on the following lines) is included in the author's Ph. D. thesis [1].

In $\S 1$ we develop the necessary structure theorems for $V$, much of which will also be found in [4], [5] and [6]; $\S 2$ gives a complete set of invariants in the improper case; while $\S 3$ treats the general case, but here we restrict ourselves to the 2 -adic numbers, the results being more complex.

Frequent use is made of the following generalization of Hensel's lemma (see [3, p. 29]): if $f(x)$ is a polynomial with coefficients in $R$ and $x_{0} \in R$ is such that $\nu\left(f\left(x_{0}\right)\right)>2 \nu\left(f^{\prime}\left(x_{0}\right)\right)$, then $f(x)$ has a root $x \in R$ with $\nu\left(x-x_{0}\right) \geqq 1$.

1. We extend the notion of order to $\alpha \in V$ by defining $\nu(\alpha)=$ $\min \nu(\alpha \cdot \beta)$, the minimum being taken over all $\beta \in V$. For each $r=$ $0,1,2, \cdots$ we denote by $V(r)$ the sublattice $\{\alpha \in V \mid \nu(\alpha) \geqq r\}$ and define $\nu_{r}(\alpha)=\min \nu(\alpha \cdot \beta)$, then minimum now being taken over all

Received August 20, 1964. 
$\beta \in V(r)$. Then

$$
\nu_{r}(\alpha) \leqq \nu_{r+1}(\alpha) \leqq \nu_{r}(\alpha)+1 \text {. }
$$

We write $V=U \oplus W$ if $U$ and $W$ are orthogonal sublattices together spanning $V$. Denote by $\left(\xi_{1}, \cdots, \xi_{s}\right)$ the sublattice spanned by $\xi_{1}, \cdots, \xi_{s} \in V$ and by $U^{\prime}$ the orthogonal complement of the sublattice $U$.

Lemma 1. Let $\xi \in V$. Then $V=(\xi) \oplus(\xi)^{\prime}$ if and only if $\nu\left(\xi^{2}\right)=$ $\nu(\xi)$.

Proof. Clearly $(\xi) \oplus(\xi)^{\prime} \subseteq V$. Take $\alpha \in V$. Then

$$
\alpha=\left(\alpha \cdot \xi / \xi^{2}\right) \xi+\left(\alpha-\left(\alpha \cdot \xi / \xi^{2}\right) \xi\right) \in(\xi) \oplus(\xi)^{\prime} .
$$

This gives the reverse inclusion. Notice that $\alpha \cdot \xi / \xi^{2} \in R$ since $\nu(\alpha \cdot \xi) \geqq$ $\nu(\xi)=\nu\left(\xi^{2}\right)$.

Lemma 2. If $\lambda, \mu \in V$ satisfy $\nu(\lambda)=\nu(\mu)=\nu(\lambda \cdot \mu)<\nu\left(\lambda^{2}\right)$ then $V=(\lambda, \mu) \oplus(\lambda, \mu)^{\prime}$.

Proof. Let $\alpha \in V$ and write $\alpha=\left(k_{1} \lambda+k_{2} \mu\right)+\left(\alpha-k_{1} \lambda-k_{2} \mu\right)$ where $k_{1}=\left((\alpha \cdot \mu)(\lambda \cdot \mu)-(\alpha \cdot \lambda) \mu^{2}\right) k_{3}^{-1}, k_{2}=\left((\alpha \cdot \lambda)(\lambda \cdot \mu)-(\alpha \cdot \mu) \lambda^{2}\right) k_{3}^{-1}$ and $k_{3}=(\lambda \cdot \mu)^{2}-\lambda^{2} \mu^{2}$. Since $\nu\left(k_{3}\right)=2 \nu(\lambda)$ it follows that $k_{1}, k_{2} \in R$. From $\lambda \cdot\left(\alpha-k_{1} \lambda-k_{2} \mu\right)=\mu \cdot\left(\alpha-k_{1} \lambda-k_{2} \mu\right)=0$ we have $\alpha \in(\lambda, \mu) \oplus(\lambda, \mu)^{\prime}$.

Lemma 3. If $V=(\xi) \oplus(\lambda, \mu)$ where $\nu(\xi)=\nu(\lambda)=\nu(\mu)=\nu(\lambda \cdot \mu)$ then there exist $\alpha, \beta, \gamma \in V$ such that $V=(\alpha) \oplus(\beta) \oplus(\gamma)$.

Proof. We may assume by Lemma 1 that $\nu\left(\lambda^{2}\right)>\nu(\lambda)$ and $\nu\left(\mu^{2}\right)>\nu(\mu)$. Let $\alpha=\xi+\lambda, \beta=\xi-\left(\xi^{2} / \lambda \cdot \mu\right) \mu$ so that $\alpha \cdot \beta=0$. Since $\nu\left(\alpha^{2}\right)=\nu(\alpha)$ and $\nu\left(\beta^{2}\right)=\nu(\beta)$ we are finished by Lemma 1 .

We can now establish the main result on the structure of $V$. Let $H_{e}$ denote a hyperbolic plane of the form $(\lambda, \mu)$ where $\lambda \cdot \mu=\pi^{e}$, $\nu\left(\lambda^{2}\right)>\nu(\lambda)=e$ and $\nu\left(\mu^{2}\right)>\nu(\mu)=e$. We call a sublattice of the form $V_{e}=H_{e} \oplus \cdots \oplus H_{e}$ improper and a sublattice of the form $V_{e}=\left(\xi_{1}\right) \oplus \cdots \oplus\left(\xi_{t}\right)$ with $\nu\left(\xi_{i}^{2}\right)=\nu\left(\xi_{i}\right)=e$, proper.

Proposition 1. (O'Meara [5]) Let $V$ be a nonsingular lattice over a local field. Then

$$
V=V_{e_{1}} \oplus V_{e_{2}} \oplus \cdots \oplus V_{e_{m}} \quad e_{1}<e_{2}<\cdots<e_{m}
$$

with the sublattices $V_{e_{i}}, 1 \leqq i \leqq m$, either proper or improper. Fur- 
thermore, $e_{i}, \operatorname{dim} V_{e_{i}}$ and the forms of the $V_{e_{i}}, 1 \leqq i \leqq m$, are invariants of $V$.

Proof. Choose $\alpha \in V$ to minimize $\nu(\alpha)$. Since the valuation is discrete there exists $\beta \in V$ such that $\nu(\alpha)=\nu(\beta)=\nu(\alpha \cdot \beta)$. If $\nu\left(\alpha^{2}\right)=$ $\nu(\alpha)$ we split off the vector $\alpha$ using Lemma 1 and if $\nu\left(\alpha^{2}\right)>\nu(\alpha)$ we split off the plane $(\alpha, \beta)$. Proceeding in this manner we obtain the stated structure for $V$, after using Lemma 3 to get the correct form for the sublattices $V_{e_{i}}$.

We now establish the invariance of $e_{i}$ and $\operatorname{dim} V_{e_{i}}, 1 \leqq i \leqq m$. Let $\xi_{1}, \cdots, \xi_{n}$ and $\eta_{1}, \cdots, \eta_{n}$ be two bases of $V$ arranged so that $\nu\left(\xi_{i}\right) \leqq$ $\nu\left(\xi_{i+1}\right)$ and $\nu\left(\eta_{i}\right) \leqq \nu\left(\eta_{i+1}\right), 1 \leqq i \leqq n-1$. It suffices to show that $\nu\left(\xi_{i}\right)=$ $\nu\left(\eta_{i}\right), 1 \leqq i \leqq n$. Let $d$ be the first discrepancy and suppose that $\nu\left(\xi_{d}\right)>\nu\left(\eta_{d}\right)=f$. Then expressing $\eta_{i}$ in terms of the $\xi_{i}$ we see that

$$
\begin{gathered}
\eta_{1}=a_{11} \xi_{1}+\cdots+a_{1 d-1} \xi_{d-1}+\alpha_{1} \\
\cdot \cdot \cdot \cdot \\
\eta_{d}=a_{d 1} \xi_{1}+\cdots+a_{d d-1} \xi_{d-1}+\alpha_{d} .
\end{gathered}
$$

In these equations $a_{i j} \in R$ and $\nu\left(\alpha_{i}\right)>f$. Eliminating the $\xi_{i}$ we see that there exist $c_{i} \in R$, with at least one $c_{i}$ a unit, such that $\sum_{i=1}^{d} c_{i} \eta_{i}=$ $\sum_{l=1}^{d} c_{i} \alpha_{i}$. But $\nu\left(\Sigma c_{i} \eta_{i}\right) \leqq f$ and $\nu\left(\Sigma c_{i} \alpha_{i}\right)>f$ gives the required contradiction.

Finally we show that the form of $V_{e_{i}}$ is invariant. Let

$$
V=V_{e_{1}} \oplus \cdots \oplus V_{e_{m}}=V_{e_{1}}^{*} \oplus \cdots \oplus V_{e_{m}}^{*}
$$

be two decompositions satisfying the conditions of the proposition and $\xi_{1}, \cdots, \xi_{n}$ the basis in the first case and $\eta_{1}, \cdots, \eta_{n}$ in the second. Suppose that $V_{e}=\left(\xi_{h}\right) \oplus \cdots \oplus\left(\xi_{k}\right)$ is proper. To prove that $V_{e}^{*}$ is proper it is sufficient to show the existence of a vector $\lambda$ orthogonal to $\eta_{1}, \cdots, \eta_{h-1}$ with $\nu\left(\lambda^{2}\right)=\nu(\lambda)=e$. If $\xi_{h}=\sum_{i=1}^{n} a_{i} \eta_{i}$ take $\lambda=\sum_{i=h}^{n} a_{i} \eta_{i}$; the required properties of $\lambda$ follow from those of $\xi_{h}$.

Lattices with the same invariants above are said to be of the same type. $A$ basis splitting $V$ into the sum of lines and planes, as in Proposition 1, is called a canonical basis.

Proposition 2. If $\alpha, \beta \in V$ satisfy $\nu(\alpha)=\nu(\beta)=\nu(\alpha \cdot \beta)=r, \nu\left(\alpha^{2}\right) \geqq$ $r+2$ and $\nu\left(\beta^{2}\right) \geqq r+1$ then there exist $\lambda, \mu \in V$ such that $V=$ $(\lambda, \mu) \oplus(\lambda, \mu)^{\prime}$ where $\lambda^{2}=\mu^{2}=0, \lambda \cdot \mu=\pi^{r}$ and $\alpha=\frac{1}{2} \pi^{-r} \alpha^{2} \lambda+\mu$.

Proof. Put $\lambda=x \alpha+\beta$ where $x$ is a root of the equation $\alpha^{2} x^{2}+2 \alpha \cdot \beta x+\beta^{2}=0$ (a root congruent to $x_{0}=-\beta^{2} / 2 \alpha \cdot \beta$ exists by 
Hensel's lemma). Then $\lambda^{2}=0$ and, multiplying by a unit if necessary, $\alpha \cdot \lambda=\pi^{r}$. Put $\mu=-\frac{1}{2} \pi^{-r} \alpha^{2} \lambda+\alpha$.

A hyperbolic plane $(\lambda, \mu)$ as in Proposition 2 is called totally isotropic.

Proposition 3. (O'Meara [6]) Let $H_{i}=\left(\lambda_{i}, \mu_{i}\right), i=1,2$, be two totally isotropic hyperbolic planes with $\nu\left(\lambda_{1}\right)=\nu\left(\lambda_{2}\right)=r$. Then an isometry $\varphi: H_{1} \rightarrow H_{2}$ extends to an isometry of $V$.

Proof. It is sufficient to show that the orthogonal complements of $H_{1}$ and $H_{2}$ are isometric. Assume first that $\lambda_{1}=\lambda_{2}$. Let $\eta_{1}, \cdots, \eta_{n-2}$ be a basis of $H_{1}^{\prime}$. Let $\zeta_{i}=\eta_{i}-\pi^{-r}\left(\eta_{i} \cdot \mu_{2}\right) \lambda_{1}, 1 \leqq i \leqq n-2$, so that we have $\zeta_{i} \cdot \zeta_{j}=\eta_{i} \cdot \eta_{j}, \zeta_{i} \cdot \lambda_{2}=\zeta_{i} \cdot \mu_{2}=0,1 \leqq i, j \leqq n-2$. Since $\zeta_{1}, \cdots, \zeta_{n-2}$ is a basis of $H_{2}^{\prime}$ this case is finished. Now we assume there exist $\alpha \in H_{1}, \beta \in H_{2}$ such that $\nu(\alpha \cdot \beta)=r$. From symmetry we may take $\nu\left(\lambda_{1} \cdot \lambda_{2}\right)=r$ and then the sublattice $\left(\lambda_{1}, \lambda_{2}\right)$ is totally isotropic by Proposition 2. By the first part we now have that $H_{1}^{\prime}$ and $H_{2}^{\prime}$ are both isometric to $\left(\lambda_{1}, \lambda_{2}\right)^{\prime}$. Finally we assume that $\nu(\alpha \cdot \beta)>r$ for all $\alpha \in H_{1}$, $\beta \in H_{2}$. The sublattice $\left(\lambda_{1}, \mu_{1}+\mu_{2}\right)$ is now totally isotropic and its orthogonal complement is isometric to $H_{1}^{\prime}$ and $H_{2}^{\prime}$ by the second part of the proof.

For $\alpha \in V$ with $\nu\left(\alpha^{2}\right)=\nu(\alpha)=r$ we define $N_{r}(\alpha)$ to be 0 if there exists $\beta \in V(r)$ such that $\alpha \cdot \beta=0$ and $\nu\left(\beta^{2}\right)=r$, and to be 1 otherwise. A vector $\alpha \in V$ is called imprimitive if $\alpha=\pi \beta$ with $\beta \in V$, otherwise it is primitive.

Proposion 4. Let $V=(\xi) \oplus(\xi)^{\prime}=(\eta) \oplus(\eta)^{\prime}$. Then there exists an isometry $\varphi$ of $V$ such that $\varphi(\xi)=\eta$ if and only if $\xi^{2}=\eta^{2}, \nu(\xi)=$ $\nu(\eta)=r$ and $N_{r}(\xi)=N_{r}(\eta)$.

Proof. For the general case see O'Meara [5, Theorems 5.1, 5.2]. See also Jones [2] for a discussion of related results in the 2 -adic case.

The rest of this section will be devoted to constructing isometric invariants of vectors.

We call $r(\neq 0)$ a critical index of $\alpha \in V$ if

$$
\nu_{r-1}(\alpha)=\nu_{r}(\alpha)<\nu_{r+1}(\alpha) ;
$$

0 is a critical index of $\alpha \in V$ if

$$
\nu(\alpha)<\nu_{1}(\alpha) \text {. }
$$

The critical indices can only be the orders of basis vectors (the $e_{i}$ of 
Proposition 1) and hence a vector has only a finite number of critical indices. They are isometric invariants.

We say that the vectors $\lambda_{1}, \cdots, \lambda_{m}$ are fully orthogonal if $V=$ $V_{1} \oplus \cdots \oplus V_{m}$ where $\lambda_{i} \in V_{i}, 1 \leqq i \leqq m$.

Proposition 5. Let $\alpha$ have critical indices at $r_{1}, r_{2}, \cdots, r_{s}$ where $r_{1}<r_{2}<\cdots<r_{s}$ and let $\nu_{r_{j}}(\alpha)=r_{j}+b_{j}$. Then

$$
r_{1}+b_{1}<r_{2}+b_{2}<\cdots<r_{s}+b_{s}, \quad b_{1}>b_{2}>\cdots>b_{s}
$$

and $\alpha=\sum_{j=1}^{s} \pi^{b j} \lambda_{j}$ where $\lambda_{j}$ are fully orthogonal, primitive vectors. Each $\lambda_{j}$ has only one critical index and

$$
\nu\left(\lambda_{j}\right)=\nu_{r_{j}}\left(\lambda_{j}\right)=r_{\jmath}, \quad 1 \leqq j \leqq s .
$$

Proof. Let $\xi_{1}, \cdots, \xi_{n}$ be a canonical basis of $V$, so that $\alpha=$ $\sum_{i=1}^{n} a_{i} \xi_{i}$. We will use induction on the number of critical indices. Let $\lambda_{1}=\pi^{-b_{1}} \Sigma^{\prime} a_{i} \xi_{i} \in V$, the sum being over those $\xi_{i}$ for which either $\nu\left(\xi_{i}\right) \leqq r_{1}$, or $\nu\left(\xi_{i}^{2}\right)=\nu\left(\xi_{i}\right)$ and $\nu\left(a_{i}\right) \geqq b_{1}$, or $\nu\left(\xi_{i}^{2}\right)>\nu\left(\xi_{i}\right)=\nu\left(\xi_{i} \cdot \xi_{i+1}\right)$ and $\nu\left(a_{i}\right) \geqq b_{1}, \nu\left(a_{i+1}\right) \geqq b_{1}$. $\lambda_{1}$ now satisfies the conditions stated and the rest of $\alpha$ is a vector with critical indices at $r_{2}, \cdots, r_{s}$.

COROLlaRY. There exist $\beta_{i} \in V, 1 \leqq i \leqq s$, such that $\beta_{i} \cdot \lambda_{j}=0$ $(i \neq j)$ and $\nu\left(\lambda_{i}\right)=\nu\left(\beta_{i}\right)=\nu\left(\beta_{i} \cdot \lambda_{i}\right)$.

The $\lambda_{j}$ are called critical components of $\alpha$. In general they are not uniquely determined. However, the $r_{j}$ and $b_{j}$ are invariant and the lengths of the $\lambda_{j}$ are invariant modulo certain powers of $\pi$.

Proposition 6. Suppose $\alpha=\sum_{j=1}^{s} \pi^{b_{j}} \lambda_{j}=\sum_{j=1}^{s} \pi^{b_{j}} \mu_{j}$ are two decompositions as in Proposion 5 with $\nu\left(\lambda_{j}\right)=\nu\left(\mu_{j}\right)=r_{j}$. Then

$$
\begin{array}{r}
\left(\pi^{-b_{t}} \sum_{j=1}^{t} \pi^{b_{j}} \mu_{j}\right)^{2} \equiv\left(\pi^{-b_{t}} \sum_{j=1}^{t} \pi^{b_{j}} \lambda_{j}\right)^{2}\left(\bmod \pi^{r_{t+1}+b_{t+1}-b_{t}}\right), \\
1 \leqq t \leqq s-1 .
\end{array}
$$

Proof. Fix $t, 1 \leqq t \leqq s-1$. By Corollary to Proposition 5 there exist $\beta_{i}$ such that $\nu\left(\beta_{i} \cdot \lambda_{i}\right)=\nu\left(\beta_{i}\right)=r_{i}, \beta_{i} \cdot \lambda_{j}=0(i \neq j)$. Then

$$
\alpha \cdot \beta_{i}=\pi^{b i} \lambda_{i} \cdot \beta_{i} \equiv \sum_{j=1}^{t} \pi^{b j} \mu_{j} \cdot \beta_{i}\left(\bmod \pi^{r_{t+1}+b_{t+1}}\right), \quad 1 \leqq i \leqq t .
$$

We can therefore find $x_{i} \in R$ such that $\nu\left(x_{i}-1\right) \geqq r_{t+1}+b_{t+1}-r_{i}-b_{i}$ and $x_{i} \lambda_{i} \cdot \beta_{i}=\pi^{-b_{i}} \sum_{j_{1}=1}^{t} \pi^{b j} \mu_{j} \cdot \beta_{i}$. This shows that the vector $\eta=$ $\sum_{j=1}^{t} \pi^{b}\left(\mu_{j}-x_{j} \lambda_{j}\right)$ is orthogonal to all the vectors $\beta_{1}, \cdots, \beta_{t}$. Furthermore 


$$
\sum_{j=1}^{t} x_{j} \pi^{b j} \lambda_{j}+\eta=\sum_{j=1}^{t} \pi^{b j} \mu_{j}
$$

We now have

$$
\alpha=\sum_{j=1}^{t} \pi^{b_{j}} \lambda_{j}+\sum_{j=t+1}^{s} \pi^{b_{j}} \lambda_{j}=\eta+\sum_{j=1}^{t} x_{j} \pi^{b_{j}} \lambda_{j}+\sum_{j=t+1}^{s} \pi^{b_{j}} \mu_{j} .
$$

Since $\nu\left(\left(x_{j}-1\right) \pi^{b j} \lambda_{j}\right) \geqq r_{t+1}+b_{t+1}$ for $1 \leqq j \leqq t$, we have

$$
\left.\pi^{-b} \iota\right) V\left(r_{t+1}+b_{t+1}-b_{t}\right) \text {. }
$$

The result is now obtained by squaring (1).

Notice that it is not necessary to assume that the $\mu_{j}$ are orthogonal. Let $D_{t}(\alpha)=\left(\pi^{-b_{t}} \sum_{l j=1}^{t} \pi^{b_{j}} \lambda_{j}\right)^{2}$ and $d_{t}(\alpha)=r_{t+1}+b_{t+1}-b_{t}$ for $1 \leqq t \leqq s-1$. We have established that $D_{t}(\alpha)\left(\bmod \pi^{a_{t}^{(\alpha)}}\right), 1 \leqq t \leqq s-1$, are invariants of the vector $\alpha$ and hence also isometric invariants.

COROLlaRY. If $V$ does not contain any vectors $\xi$ such that $\nu\left(\xi^{2}\right)=\nu(\xi)=d_{t}(\alpha)$ then $D_{t}(\alpha)$ modulo $\pi^{d_{t}(\alpha)+1}$ is actually invariant.

Under certain conditions an even stronger condition can be established.

Let $\alpha=\sum_{j=1}^{s} \pi^{b} \lambda_{j}$ be as in Proposition 5. Define $N_{r_{j}}(\alpha)$ to be 0 if there exists a $\beta \in V\left(r_{j}\right)$ such that $\beta \cdot \lambda_{j}=0$ and $\nu\left(\beta^{2}\right)=r_{j}$, otherwise let $N_{r_{j}}(\alpha)$ be 1 . $N_{r_{j}}(\alpha), 1 \leqq j \leqq s$, are independent of the choice of the critical components (left to the reader) and hence are isometric invariants.

We will show that in certain cases the above isometric invariants form a complete set.

We conclude this section with a simple observation. Let $W_{i} \subseteq V=$ $\left(\xi_{1}, \cdots, \xi_{n}\right)$ and $\operatorname{dim} W_{i}=\operatorname{dim} V, i=1,2$. Then for some $k \geqq 0$ we have $\pi^{k} V=\left(\pi^{k} \xi_{1}, \cdots, \pi^{k} \xi_{n}\right) \subseteq W_{1}$. Let $\varphi: W_{1} \rightarrow W_{2}$ be an isometry. Let $\alpha \in W_{1}$ be primitive in $W_{1}$ but imprimitive in $V$, i.e. $\alpha=\pi \beta$ where $\beta \notin W_{1}, \beta \in V$. Then if $\varphi$ is to extend to an isometry of $V$, $\varphi(\alpha)$ must be imprimitive in $V, \varphi(\alpha)=\pi \beta^{\prime}$ say, and then we must have $\varphi(\beta)=\beta^{\prime}$. Thus by considering $\pi^{k} \xi_{i} \in W_{1}$ we can determine whether $\varphi$ extends to an isometry of $V$ or not.

2. We now give a complete set of invariants for the improper case. We assume that $V$ contains no vectors $\xi$ such that $\nu\left(\xi^{2}\right)=\nu(\xi)$. Call such a lattice $V$ fully improper. 
Proposition 7. Let $\alpha$ and $\beta$ be vectors with one critical index, at $r$. Then there exists an isometry $\varphi$ of $V$ such that $\varphi(\alpha)=\beta$ if and only if $\alpha^{2}=\beta^{2}$ and $\nu(\alpha)=\nu(\beta)$.

Proof. We may assume $\alpha$ and $\beta$ are primitive so that $\nu(\alpha)=$ $\nu(\beta)=r$. If $\nu(\alpha)=\nu(\alpha \cdot \beta)$ then by Lemma $2 V=(\alpha, \beta) \oplus(\alpha, \beta)^{\prime}$. Define an isometry $\varphi$ by $\varphi(\alpha)=\beta, \varphi(\beta)=\alpha$ and identity on $(\alpha, \beta)^{\prime}$. We may therefore assume $\nu(\alpha \cdot \beta)>\nu(\alpha)$. Now choose $\rho \in V(r)$ such that $\nu(\alpha \cdot \rho)=\nu(\beta \cdot \rho)=\nu(\alpha)=\nu(\rho)$. If $\nu\left(\rho^{2}\right)>\nu(\rho)+1$, then $(\alpha, \rho)$ and $(\beta, \rho)$ are totally isotropic, and we are finished by Proposition 3. If $\nu\left(\rho^{2}\right)=\nu(\rho)+1$, put $\gamma=x \rho+\alpha$ where $x \rho^{2}+2 \rho \cdot \alpha=0$, so that $\alpha^{2}=$ $\beta^{2}=\gamma^{2}$. Now as above, we can find isometries $\varphi_{1}, \varphi_{2}$ such that $\varphi_{1}(\alpha)=$ $\gamma, \varphi_{2}(\gamma)=\beta$. Put $\varphi=\varphi_{2} \varphi_{1}$.

For vectors with more than one critical index we use the invariants of Proposition 6 to adjust the lengths of the critical components and then split them off in orthogonal sublattices. The proof is then complete by induction. We need first another result.

Proposition 8. Let $V=\left(\lambda_{1}, \eta\right) \oplus\left(\lambda_{2}, \xi\right)$ where $\nu\left(\lambda_{1}\right)=\nu\left(\lambda_{1} \cdot \eta\right)=$ $\nu(\eta)=r_{1}$ and $\nu\left(\lambda_{2}\right)=\nu(\xi)=\nu\left(\lambda_{2} \cdot \xi\right)=r_{2}$. If $\alpha=\pi^{c} \lambda_{1}+\lambda_{2}$ where $c \geqq 1$, and if $\nu(y) \geqq r_{2}-r_{1}-c>0$, then there exists an isometry $\varphi$ of $V$ such that

$$
\varphi(\alpha)=\beta=\pi^{c}\left(\lambda_{1}+y \eta\right)+z \xi-\lambda_{2}
$$

for some $z \in R$.

Proof. Let $z \in R$ be a root of the equation

$$
\frac{1}{2} z^{2} \xi^{2}-z \lambda_{2} \cdot \xi+\pi^{2 c} y\left(\lambda_{1} \cdot \eta+\frac{y}{2} \eta^{2}\right)=0 .
$$

Then $\nu(z) \geqq c$ and $\alpha^{2}=\beta^{2}$. Put

$$
\begin{aligned}
& \gamma_{1}=\pi^{c}\left(\lambda_{1}+\frac{1}{2} y \eta\right)+\frac{1}{2} z \xi, \\
& \gamma_{2}=\frac{1}{2} \pi^{c} y \eta+\frac{1}{2} z \xi-\lambda_{2}, \\
& \gamma_{3}=\eta-\left(\frac{\eta^{2}}{\eta \cdot \lambda_{1}} \lambda_{1}\right) \lambda_{1}, \\
& \gamma_{4}=\xi-\left(\frac{\xi^{2}}{\xi \cdot \lambda_{2}} \lambda_{2}\right) \lambda_{2} .
\end{aligned}
$$

It follows that $\gamma_{1}-\gamma_{2}=\alpha, \gamma_{1}+\gamma_{2}=\beta$ and $\gamma_{1} \cdot \gamma_{2}=\gamma_{1} \cdot \gamma_{4}=\gamma_{2} \cdot \gamma_{3}=$ 
$\gamma_{3} \cdot \gamma_{4}=0$. Define an isometry $\varphi$ on the sublattice $\left(\gamma_{1}, \gamma_{2}, \gamma_{3}, \gamma_{4}\right)$ of $V$ by

$$
\begin{array}{ll}
\varphi\left(\gamma_{1}\right)=\gamma_{1}, \quad & \varphi\left(\gamma_{2}\right)=-\gamma_{2}, \\
\varphi\left(\gamma_{3}\right)=\gamma_{3}, & \varphi\left(\gamma_{4}\right)=-\gamma_{4} .
\end{array}
$$

By considering the vectors in this sublattice that are imprimitive in $V$ we see that $\varphi$ extends uniquely to an isometry of $V$. Since $\varphi(\alpha)=$ $\beta$ we are finished.

Proposition 9. Let $V$ be a fully improper lattice. Take $\alpha, \beta \in V$. Then there exists an isometry $\varphi$ of $V$ such that $\varphi(\alpha)=\beta$ if and only if

1. $\alpha^{2}=\beta^{2}$

2. $\alpha$ and $\beta$ have the same critical indices $r_{1}, \cdots, r_{s}$

3. $\nu_{r_{i}}(\alpha)=\nu_{r_{i}}(\beta)=r_{i}+b_{i}$

$1 \leqq i \leqq s$

4. $D_{t}(\alpha) \equiv D_{t}(\beta)\left(\bmod \pi^{d_{t}(\alpha)+1}\right)$

$1 \leqq t \leqq s-1$.

Proof. The necessity of the conditions follows from $\S 1$. Notice that the invariant $N_{r_{i}}(\alpha)$ is always 1 in this case. For the sufficiency we use induction on $s$. The case $s=1$ is Proposition 7. Take $s>1$. Let $\alpha=\sum_{i=1}^{s} \pi^{b i} \lambda_{i}$ and $\beta=\sum_{i=1}^{s} \pi^{b} i \mu_{i}$ be decompositions into fully orthogonal critical components. Let $\eta$ be such that $\nu(\eta)=\nu\left(\eta \cdot \lambda_{1}\right)=\nu\left(\lambda_{1}\right)$ and $\eta \cdot \lambda_{i}=0(i \neq 1)$. Then from 4 , with $t=1$, we have

$$
\lambda_{1}^{2} \equiv \mu_{1}^{2}\left(\bmod \pi^{r_{2}+b_{2}-b_{1}+1}\right) \text {. }
$$

Take $y \in R$ such that $\left(\lambda_{1}+y \eta\right)^{2}=\mu_{1}^{2}$; this equation has a solution $y$ such that $\nu(y) \geqq r_{2}+b_{2}-r_{1}-b_{1}$. By Proposition 8, taking $c=$ $b_{1}-b_{2} \geqq 1$, there exists an isometry of $V$ mapping $\pi^{b_{1}} \lambda_{1}+\pi^{b_{2}} \lambda_{2}$ into $\pi^{b_{1}}\left(\lambda_{1}+y \eta\right)+\pi^{b_{2}}\left(z \xi-\lambda_{2}\right)$, i.e. mapping $\alpha$ into a vector $\alpha_{1}$ whose first critical component has the same length as the first critical component of $\beta$. We now split off the first critical component of $\alpha_{1}$ into a binary sublattice. We then proceed to adjust the lengths of the remaining critical components of $\alpha$.

In this way we can map $\alpha=\Sigma \pi^{b i} \lambda_{i}$ into a vector $\alpha^{*}=\Sigma \pi^{b i} \lambda_{i}^{*}$ with $\lambda_{i}^{* 2}=\mu_{i}^{2}, 1 \leqq i \leqq s$. Those $\lambda_{i}^{*}, \mu_{i}$ satisfying $\nu\left(\mu_{i}^{2}\right) \geqq \nu\left(\mu_{i}\right)+2$ can be imbedded in totally isotropic, binary lattices and mapped into each other using Propositions 2 and 3. We may therefore assume that $\nu\left(\mu_{i}^{2}\right)=\nu\left(\mu_{i}\right)+1$ for all $i$.

We now restrict ourselves to studying $\alpha=\Sigma \pi^{b_{i}} \lambda_{i}$ and $\beta=\Sigma \pi^{b_{i}} \mu_{i}$ with $\lambda_{i}^{2}=\mu_{i}^{2}$ and $\nu\left(\lambda_{i}^{2}\right)=\nu\left(\lambda_{i}\right)+1$. By Proposition 7 we may assume $\mu_{1}=\lambda_{1}$ after applying an isometry to $\alpha$. Take $\rho \in V$ such that $\nu\left(\mu_{1} \cdot \rho\right)=\nu\left(\mu_{1}\right)=\nu(\rho)$ and $\rho \cdot \mu_{i}=0,2 \leqq i \leqq s$. Using Lemma 2 we can split off $\left(\mu_{1}, \rho\right)$. However, we will not have $\rho \cdot \lambda_{i}=0$ in general. 
We apply a further isometry $\theta$ to $\alpha$ keeping $\lambda_{1}$ fixed and mapping $\lambda_{i}$, $2 \leqq i \leqq s$, into vectors orthogonal to $\rho$. Then splitting off $\left(\lambda_{1}, \rho\right)$ we are finished by induction.

We construct $\theta$ as follows. Let $V=\left(\lambda_{1}, \eta_{1}\right) \oplus\left(\lambda_{2}, \xi_{1}\right) \oplus U$ where $\lambda_{i}, 3 \leqq i \leqq s$, are in the sublattice $U$. We may assume that $\nu\left(\eta_{1}^{2}\right)=$ $\nu\left(\eta_{1}\right)+1$ and $\nu\left(\xi_{1}^{2}\right)=\nu\left(\xi_{1}\right)+1$, for otherwise we have totally isotropic, binary lattices and these may be concelled. Thus we may assume $\xi_{1}^{2}=\lambda_{2}^{2}$. Let

$$
\lambda_{2}^{\prime}=x \pi^{r_{2}-r_{1}}\left(\lambda_{1}-\left(\frac{\lambda_{1}^{2}}{\lambda_{1} \cdot \eta_{1}} \eta_{1}\right)+y \lambda_{2}+\xi_{1},\right.
$$

where $x$ and $y$ are chosen so that $\lambda_{2}^{\prime 2}=\lambda_{2}^{2}$ and $\lambda_{2}^{\prime} \cdot \rho=0$ (the equations for $x$ and $y$ have solutions in $R$ ). Then $\lambda_{2}^{\prime} \cdot \lambda_{1}=0$ and $\nu\left(\lambda_{2}^{\prime} \cdot \lambda_{2}\right)=\nu\left(\lambda_{2}\right)$. Thus $V=\left(\lambda_{1}, \gamma\right) \oplus\left(\lambda_{2}, \lambda_{2}^{\prime}\right) \oplus U$ for some $\gamma \in V$. We now take an isometry mapping $\lambda_{2}$ into $\lambda_{2}^{\prime}$ and acting as the identity on $\left(\lambda_{2}, \lambda_{2}^{\prime}\right)^{\prime}$. Similarly we can map $\lambda_{i}, 3 \leqq i \leqq s$, into vectors orthogonal to $\rho$.

3. We now consider the general case but restrict ourselves to $R$ being the ring of 2 -adic integers.

Proposition 10. Let $\alpha$ and $\beta$ be vectors with one critical index, at $r$. Then there exists an isometry $\varphi$ of $V$ such that $\varphi(\alpha)=\beta$ if and only if $\alpha^{2}=\beta^{2}, \nu(\alpha)=\nu(\beta)$ and $N_{r}(\alpha)=N_{r}(\beta)$.

Proof. We may assume $\alpha$ and $\beta$ primitive so that $\nu(\alpha)=\nu(\beta)=r$. If $\nu\left(\alpha^{2}\right)=\nu(\alpha)$ we are finished by Proposition 4. Assume $\nu\left(\alpha^{2}\right)>\nu(\alpha)$. As in Proposition 7 we take $\rho \in V(r)$ such that $\nu(\alpha \cdot \rho)=\nu(\beta \cdot \rho)=\nu(\alpha)$. We need only consider the case $\nu\left(\rho^{2}\right)=\nu(\rho)$. Then we may assume that $\alpha=x \rho+\eta, \beta=\rho+\xi$ with $\eta \cdot \rho=\xi \cdot \rho=0$. We now wish to find $\lambda, \mu \in V$ such that $\alpha=\lambda+\mu, \lambda \cdot \mu=0$ and $\lambda^{2}=\rho^{2}$. The necessary equations can be solved, but only because $\nu(x)=0$ so that for the 2 -adics $\nu\left(x^{2}-1\right) \geqq 3$. We then construct an isometry $\varphi$ such that $\varphi(\lambda)=\rho$ and $\varphi(\mu)=\xi$ using Proposition 4.

This result need not be true if we go beyond the 2-adics; further invariants are necessary as is shown by an example latter.

To treat vectors with more than one critical component we must establish some results analogous to Proposition 8. The situation is now more complex and the particular invariants depend on the structure of $V$. For there to exist an isometry mapping $\alpha$ into $\beta$ we need the following invariants:

(i) $\alpha^{2}=\beta^{2}$

(ii) $\alpha$ and $\beta$ have the same critical indices $r_{1}, \cdots, r_{s}$ 

(iii) $N_{r_{i}}(\alpha)=N_{r_{i}}(\beta)$,
$1 \leqq i \leqq s$
(iv) $\nu_{r_{i}}(\alpha)=\nu_{r_{i}}(\beta)=r_{i}+b_{i}$
$1 \leqq i \leqq s$
(v) $D_{t}(\alpha) \equiv D_{t}(\beta)\left(\bmod \pi^{a_{t}(\alpha)+f t}\right)$,
$1 \leqq t \leqq s-1$,

where $f_{t}=0,1$ or 2 depending on the structure of $V$.

These conditions, with $f_{t}=2$, are always sufficient, but usually not necessary. The conditions with $f_{t}=1$ are necessary, provided there do not exist any $\xi_{t} \in V$ satisfying $\nu\left(\xi_{t}^{2}\right)=\nu\left(\xi_{t}\right)=d_{t}(\alpha)$ for $1 \leqq t \leqq s-1$, and also sufficient provided further that $b_{i-1} \neq b_{i}+1$ or $r_{i+1}+b_{i+1} \neq$ $r_{i}+b_{i}+1$ for any $i$. The proofs of these remarks are similar to the proof of Proposition 9 using results analogous to Proposition 8 to adjust the lengths of the critical components.

Proposition 11. Let $V=\left(\lambda_{1}\right) \oplus\left(\lambda_{2}\right)$ where $\nu\left(\lambda_{1}\right)=r_{1}, \nu\left(\lambda_{2}\right)=r_{2}$ and let $c \geqq 1$ and $r_{2}-r_{1}-c \geqq 1$. If $\alpha=\pi^{c} \lambda_{1}+\lambda_{2}$ then there exists an isometry $\varphi$ of $V$ such that

$$
\varphi(\alpha)=\beta=\pi^{c} x \lambda_{1}-y \lambda_{2}
$$

for some $y \in R$, provided either

or

(a) $\nu(x-1) \geqq r_{2}-r_{1}-c+1$,

(b) $\nu(x-1) \geqq r_{2}-r_{1}-c \geqq 2, c \geqq 2$.

Proof. Let $y$ be a root of the equation

$$
y^{2}=1+\pi^{2 c}\left(1-x^{2}\right) \frac{\lambda_{1}^{2}}{\lambda_{2}^{2}}, \quad y \equiv 1(\bmod 4) .
$$

This equation has a root $y \in R$ by (a) or (b) using Hensel's lemma. Put

$$
\gamma_{1}=\frac{1}{2} \pi^{c}(1+x) \lambda_{1}+\frac{1}{2}(1-y) \lambda_{2}
$$

and

$$
\gamma_{2}=\frac{1}{2} \pi^{c}(1-x) \lambda_{1}+\frac{1}{2}(1+y) \lambda_{2}
$$

Then $\gamma_{1}+\gamma_{2}=\alpha, \gamma_{1}-\gamma_{2}=\beta$ and $\gamma_{1} \cdot \gamma_{2}=0$. For (a) we have $\nu\left(\gamma_{2}^{2}\right)=$ $\nu\left(\gamma_{2}\right)$, so that $V=\left(\gamma_{2}\right) \oplus\left(\gamma_{2}\right)^{\prime}$ and we take $\varphi$ such that $\varphi\left(\gamma_{2}\right)=-\gamma_{2}$ and $\varphi$ is the identity on $\left(\gamma_{2}\right)^{\prime}$. Then $\varphi(\alpha)=\beta$. For (b) we take the isometry on $\left(\gamma_{1}, \gamma_{2}\right)$ defined by $\varphi\left(\gamma_{1}\right)=\gamma_{1}, \varphi\left(\gamma_{2}\right)=-\gamma_{2}$ and extend this to $V$ through the imprimitive vectors as in Proposition 8.

Proposition 12. Let $V=\left(\lambda_{1}\right) \oplus\left(\lambda_{2}, \xi\right)$ where $\nu\left(\lambda_{1}\right)=r_{1}, \nu\left(\lambda_{2}\right)=$ $\nu(\xi)=\nu\left(\lambda_{2} \cdot \xi\right)=r_{2}, \nu\left(\lambda_{2}^{2}\right)>\nu\left(\lambda_{2}\right)$ and $\nu\left(\xi^{2}\right)>\nu(\xi)$. If $\alpha=\pi^{c} \lambda_{1}+\lambda_{2}$ with 
$c \geqq 1$, then there exists an isometry $\varphi$ of $V$ such that

$$
\varphi(\alpha)=\beta=\pi^{c} x \lambda_{1}+y \xi-\lambda_{2}
$$

for some $y \in R$, provided either $\nu(x-1) \geqq r_{2}-r_{1}-c+1$ or $\nu(x-1) \geqq$ $r_{2}-r_{1}-c \geqq 2$.

Proposition 13. Let $V=\left(\lambda_{1}, \eta\right) \oplus\left(\lambda_{2}\right)$ where $\nu\left(\lambda_{1}\right)=\nu(\eta)=$ $\nu\left(\lambda_{1} \cdot \eta\right)=r_{1}, \nu\left(\lambda_{1}^{2}\right)>\nu\left(\lambda_{1}\right), \nu\left(\eta^{2}\right)>\nu(\eta)$ and $\nu\left(\lambda_{2}\right)=r_{2}$. If $\alpha=\pi^{c} \lambda_{1}+\lambda_{2}$ with $c \geqq 1$ and $r_{2}-r_{1}-c>0$, then there exists an isometry $\varphi$ of $V$ such that

$$
\varphi(\alpha)=\beta=\pi^{c}\left(\lambda_{1}+y \eta\right)-z \lambda_{2}
$$

for some $z \in R$, provided that either $\nu(y) \geqq r_{2}-r_{1}-c+1$ or $\nu(y) \geqq$ $r_{2}-r_{1}-c$ and $c \geqq 2$.

The proofs of Propositions 12 and 13 are similar to those of Propositions 8 and 11. These results now enable us to establish the results stated for isometric invariants in the 2 -adic case above.

Suppose that $\alpha=\sum_{i=1}^{s} \pi^{b} \lambda_{i}$ and $\beta=\sum_{i=1}^{s} \pi^{b i} \mu_{i}$ satisfy conditions (i)-(v) above (in some case of $f_{t}$ ). If $\nu\left(\lambda_{1}^{2}\right)=\nu\left(\lambda_{1}\right)$, then from (v) $\lambda_{1}^{2} \equiv$ $\mu_{1}^{2}\left(\bmod \pi^{d_{1}(\alpha)+f_{1}}\right)$ so that $\lambda_{1}^{2}=x \mu_{1}^{2}$ with $\nu(x-1) \geqq d_{1}(\alpha)+f_{1}-r_{1}$. Provided that $d_{1}(\alpha)+f_{1}-r_{1} \geqq 3$, we may take $\lambda_{1}^{2}=x^{2} \mu_{1}^{2}$ with $\nu(x-1) \geqq$ $d_{1}(\alpha)+f_{1}-r_{1}-1$. Then, applying an isometry as in Proposition 11 or 12, we may assume that $\lambda_{1}=\mu_{1}$, etc. Likewise, if $\nu\left(\lambda_{1}^{2}\right)>\nu\left(\lambda_{1}\right)$, we use suitable combinations of the methods given above.

The presence of vectors satisfying $\nu\left(\xi_{t}^{2}\right)=\nu\left(\xi_{t}\right)=d_{t}(\alpha)$ for certain $t$ enable us to use the weakest form of (v) with $f_{t}=0$, but because of the proliferation of cases we proceed no further with this.

To extend the above beyond the 2-adics requires further invariants as is shown by the following example:

$$
\begin{gathered}
V=\left(\xi_{1}\right) \oplus\left(\xi_{2}\right) \oplus\left(\xi_{3}\right) \\
\xi_{1}^{2}=\xi_{2}^{2}=1, \quad \xi_{3}^{2}=2 \\
\alpha=\xi_{1}+\xi_{2}, \quad \beta=a \xi_{1}+a \xi_{2}+b \xi_{3}
\end{gathered}
$$

where the residue class field has four elements (i.e. $a b=1, a^{2}=b$, $b^{2}=a$ and $\left.a+b=1\right)$. Although (i) $-(\mathrm{v})$ are satisfied no isometry of $V$ maps $\alpha$ into $\beta$. To show this look at the conditions imposed upon the image of $\xi_{3}$ modulo 4 . 


\section{REFERENCES}

1. D. G. James, The extension of isometries of sublattices over local fields, M.I.T. Ph. D. Thesis, 1963.

2. B. W. Jones, The arithmetic theory of quadratic forms, Carus Monographs, No. 10, Mathematical Association of America, 1950.

3. S. Lang, Algebraic Numbers, Addison-Wesley, 1964.

4. O. T. O'Meara, Introduction to quadratic forms, Springer-Verlag, 1963.

5. - Quadratic forms over local fields, Amer. J. Math. 77 (1955), 87-116.

6. Witt's theorem and the isometry of lattices, Proc. Am. Math. Soc. 7 (1956), 9-22.

7. S. Rosenzweig, An analogy of Witt's theorem for modules over the ring of p-adic integers, M.I.T. Ph. D. Thesis, 1958.

UNIVERSITY OF AUCKLAND 


\section{PACIFIC JOURNAL OF MATHEMATICS}

\section{EDITORS}

\author{
H. Samelson \\ Stanford University \\ Stanford, California \\ R. M. Blumenthal \\ University of Washington \\ Seattle, Washington 98105
}

\author{
J. Dugundu \\ University of Southern California \\ Los Angeles, California 90007 \\ *Richard Arens \\ University of California \\ Los Angeles, California 90024
}

\section{ASSOCIATE EDITORS}
E. F. BeCKENBACH
B. H. NeUMANN
F. WOLF
K. YosidA

\section{SUPPORTING INSTITUTIONS}

\author{
UNIVERSITY OF BRITISH COLUMBIA \\ CALIFORNIA INSTITUTE OF TECHNOLOGY \\ UNIVERSITY OF CALIFORNIA \\ MONTANA STATE UNIVERSITY \\ UNIVERSITY OF NEVADA \\ NEW MEXICO STATE UNIVERSITY \\ OREGON STATE UNIVERSITY \\ UNIVERSITY OF OREGON \\ OSAKA UNIVERSITY \\ UNIVERSITY OF SOUTHERN CALIFORNIA
}

\author{
STANFORD UNIVERSITY \\ UNIVERSITY OF TOKYO \\ UNIVERSITY OF UTAH \\ WASHINGTON STATE UNIVERSITY \\ UNIVERSITY OF WASHINGTON \\ * * * * \\ AMERICAN MATHEMATICAL SOCIETY \\ CALIFORNIA RESEARCH CORPORATION \\ SPACE TECHNOLOGY LABORATORIES \\ NAVAL ORDNANCE TEST STATION
}

Mathematical papers intended for publication in the Pacific Journal of Mathematics should by typewritten (double spaced). The first paragraph or two must be capable of being used separately as a synopsis of the entire paper. It should not contain references to the bibliography. No separate author's resumé is required. Manuscripts may be sent to any one of the four editors. All other communications to the editors should be addressed to the managing editor, Richard Arens, at the University of California, Los Angeles, California 90024.

50 reprints per author of each article are furnished free of charge; additional copies may be obtained at cost in multiples of 50 .

The Pacific Journal of Mathematics is published quarterly, in March, June, September, and December. Effective with Volume 13 the price per volume (4 numbers) is $\$ 18.00$; single issues, $\$ 5.00$. Special price for current issues to individual faculty members of supporting institutions and to individual members of the American Mathematical Society: $\$ 8.00$ per volume; single issues $\$ 2.50$. Back numbers are available.

Subscriptions, orders for back numbers, and changes of address should be sent to Pacific Journal of Mathematics, 103 Highland Boulevard, Berkeley 8, California.

Printed at Kokusai Bunken Insatsusha (International Academic Printing Co., Ltd.), No. 6, 2-chome, Fujimi-cho, Chiyoda-ku, Tokyo, Japan.

PUBLISHED BY PACIFIC JOURNAL OF MATHEMATICS, A NON-PROFIT CORPORATION

The Supporting Institutions listed above contribute to the cost of publication of this Journal, but they are not owners or publishers and have no responsibility for its content or policies.

* Basil Gordon, Acting Managing Editor until February 1, 1966. 


\section{Pacific Journal of Mathematics}

\section{Vol. 15, No. $3 \quad$ November, 1965}

David R. Arterburn and Robert James Whitley, Projections in the space of

bounded linear operators .................................

Robert McCallum Blumenthal, Joram Lindenstrauss and Robert Ralph Phelps,

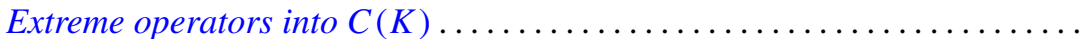

L. Carlitz, A note on multiple exponential sums ................... 757

Joseph A. Cima, A nonnormal Blaschke-quotient .................... 767

Paul Civin and Bertram Yood, Lie and Jordan structures in Banach algebras . . . 775

Luther Elic Claborn, Dedekind domains: Overrings and semi-prime

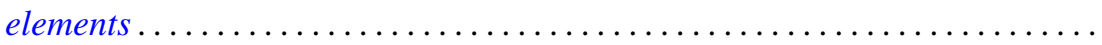

799

Luther Elic Claborn, Note generalizing a result of Samuel's .............. 805

George Bernard Dantzig, E. Eisenberg and Richard Warren Cottle, Symmetric

dual nonlinear programs ................................... 809

Philip J. Davis, Simple quadratures in the complex plane ............... 813

Edward Richard Fadell, On a coincidence theorem of F. B. Fuller ............ 825

Delbert Ray Fulkerson and Oliver Gross, Incidence matrices and interval

graphs ........................................ 835

Larry Charles Grove, Tensor products over $H^{*}$-algebras ..................

Deborah Tepper Haimo, $L^{2}$ expansions in terms of generalized heat polynomials

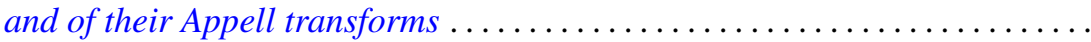

I. Martin (Irving) Isaacs and Donald Steven Passman, A chardcterization of groups in terms of the degrees of their characters ..........

Donald Gordon James, Integral invariants for vectors over local fields........ 905

Fred Krakowski, A remark on the lemma of Gauss ................... 917

Marvin David Marcus and H. Minc, A subdeterminant inequality ........... 921

Kevin Mor McCrimmon, Norms and noncommutative Jordan algebras ........ 925

Donald Earl Myers, Topologies for Laplace transform spaces ............... 957

Olav Njstad, On some classes of nearly open sets .................... 961

Milton Philip Olson, A characterization of conditional probability ........... 971

Barbara Osofsky, A counter-example to a lemma of Skornjakov .............. 985

Sidney Charles Port, Ratio limit theorems for Markov chains ............... 989

George A. Reid, A generalisation of $W^{*}$-algebras ...................... 1019

Robert Wells Ritchie, Classes of recursive functions based on Ackermann's

function ........................................... 1027

Thomas Lawrence Sherman, Properties of solutions of $n$th order linear

differential equations ........................................ 1045

Ernst Snapper, Inflation and deflation for all dimensions . .............. 1061

Kondagunta Sundaresan, On the strict and uniform convexity of certain Banach

spaces............................................. 1083

Frank J. Wagner, Maximal convex filters in a locally convex space .......... 1087

Joseph Albert Wolf, Translation-invariant function algebras on compact 